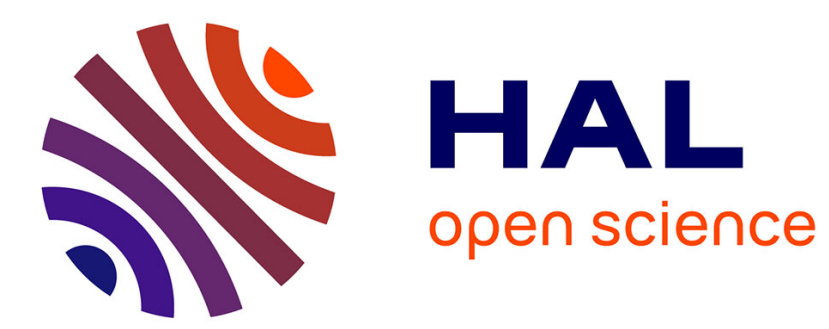

\title{
Dynamique des nombres et physique des oscillateurs Jacky Cresson
}

\section{To cite this version:}

Jacky Cresson. Dynamique des nombres et physique des oscillateurs. 2005. hal-00008872

\section{HAL Id: hal-00008872}

\section{https://hal.science/hal-00008872}

Preprint submitted on 19 Sep 2005

HAL is a multi-disciplinary open access archive for the deposit and dissemination of scientific research documents, whether they are published or not. The documents may come from teaching and research institutions in France or abroad, or from public or private research centers.
L'archive ouverte pluridisciplinaire HAL, est destinée au dépôt et à la diffusion de documents scientifiques de niveau recherche, publiés ou non, émanant des établissements d'enseignement et de recherche français ou étrangers, des laboratoires publics ou privés. 


\title{
DYNAMIQUE DES NOMBRES ET PHYSIQUE DES OSCILLATEURS
}

\author{
par
}

Jacky CRESSON

\section{Table des matières}

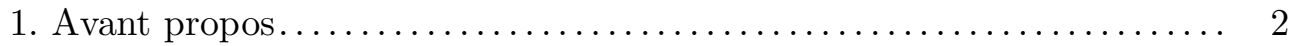

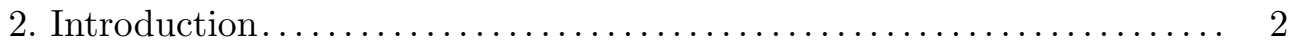

3. Spectre de fréquences expérimental. .......................... 4

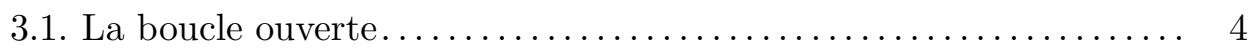

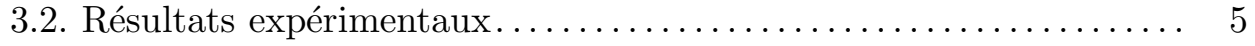

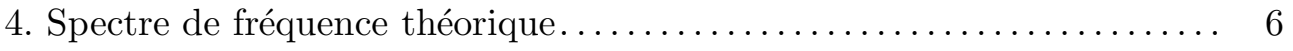

4.1. Formalisation et hypothèse diophantienne. ............... 6

4.2. Principaux résultats. .................................... 7

4.3. Confirmation expérimentale. ............................ 8

4.4. Exploration de la condition diophantienne. ................ 9

5. Espaces de résolution: aspects géométriques. ................... 9

5.1. Géométrie des fractions continues. ...................... 9

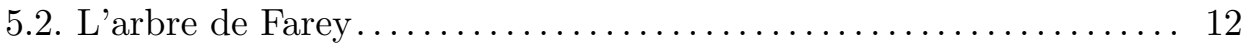

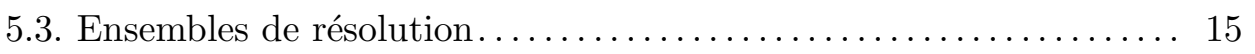

6. Espaces de résolution: aspects dynamiques .................... 15

6.1. Système dynamique de résolution . ....................... 15

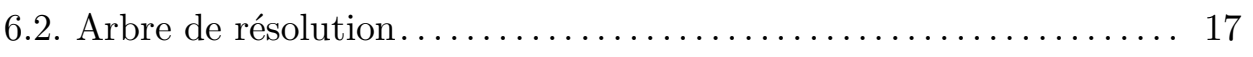

7. Construction dynamique et théorème de structure $\ldots \ldots \ldots \ldots \ldots \ldots 17$

7.1. Sur les zônes d'accrochages. ........................... 19

7.2. Bassin d'attraction d'un rationne]...................... 20

8. Approche dynamique du spectre des fréquences. ............... 21

8.1. Dynamique des fractions continues. ................... 21

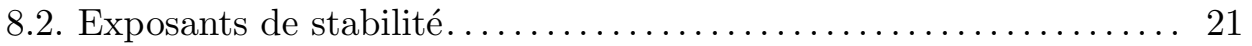

8.3. Instabilité au voisinage des résonances $\ldots \ldots \ldots \ldots \ldots \ldots \ldots \ldots 22$ 
9. Réalité ou artefact ?...................................... 23

Références.............................................. 24

\section{Avant propos}

Le but de cet article est de présenté un exemple d'intéraction entre la théorie des nombres et la physique expérimentale. J'insiste sur l'aspect expérimental, car il n'est pas rare de trouver des applications de la théorie des nombres en physique théorique, ou plus généralement en physique mathématique (voir [22]). L'intérêt est donc d'avoir sous la main un dispositif expérimental qui permet de visualiser directement cette intéraction.

Les mathématiques sous-jacente à cette intéraction sont élémentaires (essentiellement, elles tournent autour des fractions continues). J'ai néanmoins décidé d'en faire une présentation complète pour au moins deux raisons:

- La première est que l'approche que nous allons emprunter n'est pas l'approche standard du sujet, et suggère des interprétations et des notions nouvelles.

- La seconde est que ce texte est destiné à une communauté beaucoup plus large que les mathématiciens, qui n'est pas spécialement familière des notions mathématiques utilisées.

\section{Introduction}

Les systèmes de communications nécessitent la mise au point de circuits électroniques permettant de convertir, moduler et détecter des fréquences. Par exemple, la propagation des ondes radio est plus efficace pour des fréquences élevées. On cherche donc à transformer le signal afin de faire porter l'information initiale par un signal haute fréquence. Ce procédé a de plus l'avantage de réduire la taille des antennes nécessaires à la reception (voir 21],p.487). La boucle ouverte est le composant électronique de base le plus répendu pour effectuer des modification de fréquences. Il est fondé sur un mélangeur qui théoriquement "fait" le produit de deux signaux.

Récemment, une série d'expériences destinées à étudier le bruit en $1 / f$, menées par michel Planat au LPMO, ont conduit à un renouvellement de notre comprehension du mélangeur et de la boucle ouverte. Ce renouvellement est dû en partie à la grande 
précision des mesures du spectre des fréquences et d'amplitudes du signal de sortie.

Les principales nouveautés dans l'analyse du mélangeur et de la boucle ouverte sont les suivantes :

- Le spectre des fréquences est gouverné par une analyse de type diophantienne.

- Il existe une résolution minimale (en temps et espace), intrinsèque au système, structurant l'espace des fréquences via l'analyse diophantienne du point précédent.

Le résultat principal de cet article est un théorème abstrait permettant de prédire la structure du spectre expérimentale de fréquences.

Les points 1 et 2 demandent l'introduction d'espaces de résolution (appelés espaces de résolution arithmétiques dans [3]). Ils prennent en compte l'aspect diophantiens et les contraintes de résolution minimale en espace et temps.

La nécessité d'avoir une information sur l'approximation diophantienne des nombres réels conduit naturellement aux fractions continues. On en donne une présentation originale via les deux opérations élémentaires $x \mapsto x+1$ et $x \mapsto 1 / x$. Notamment, on obtient une représentation nouvelle, à notre connaissance, de l'arbre de Farey. Ce choix de construction et de représentation des nombres est dicté par la nécessité d'avoir une traduction aussi simple que possible des contraintes de résolution.

La contrainte de résolution en espace s'interpréte comme l'existence d'un entier, noté $a_{\text {max }}$, au delà duquel, les nombres sont identifiés avec l'infini. On introduit ainsi une structure d'échelle naturelle, dans l'ensemble précédent, en faisant apparaître des zônes de blocage (ou d'accrochage) des nombres rationnels, des zônes de transitions vers les zônes de blocage et enfin des zônes d'instabilité, correspondant à des irrationnels. Concrétement, on retrouve l'ensemble des fractions continues à quotients partiels bornés par $a_{\max }$. On en donne une construction originale faisant intervenir un système dynamique naturel sur l'ensemble des fractions continues et conduisant à une dynamique des nombres. Cette dynamique n'est apparente que lorsque $a_{\max }$ est fini. On montre ainsi qu'il existe, dès qu'une contrainte de résolution est fixée, une hiérarchie naturelle des nombres, hiérarchie qui disparait si on regarde $\mathbb{R}$ tout entier.

La contrainte en temps, se traduit par l'existence d'une borne $n_{\max }$ à la longueur des fractions continues. Autrement dit, le système ne peut pas "descendre" dans le développement en fraction continue d'un nombre indéfiniment. On introduit alors une 
notion de zône floue, qui représente des endroits ou l'analyse du système ne donne aucune information, les nombres à analyser ayant un développement en fraction continue trop grand. Autrement dit, le système fait bien quelque chose, mais il est impossible de savoir quoi.

Le spectre des amplitudes ne se laisse pas aussi facilement capturer. Il n'existe pas pour le moment un analogue du théorème de structure.

\section{Spectre de fréquences expérimental}

On présente le modèle de mélangeur et de filtre passe-bas qui nous servira dans le reste de l'article. Nous décrivons le spectre des fréquences expérimental obtenu. Nous formulons ensuite notre approche du spectre des fréquences et l'hypothèse principale de ce travail, à savoir que la boucle ouverte "fait" de l'approximation diophantienne des fréquences du signal.

3.1. La boucle ouverte. - La boucle ouverte ou montage superhétérodyne découvert par Armstrong et Schottky en 1924 permet d'étudier un signal, appelé signal de référence et noté $s_{0}(t)$, à partir d'un signal connu noté $s_{1}(t)$. La fréquence de l'oscillateur de référence est notée $f_{0}(t)$. Le signal connu est produit par un oscillateur dit local de fréquence $f_{1}$.

La boucle ouverte est composée d'un mélangeur qui doit multiplier les deux signaux et d'un filtre dit passe-bas de fréquence de coupure $f_{c}$, qui doit couper les fréquences au dessus de $f_{c}$. On a donc le dispositif suivant:

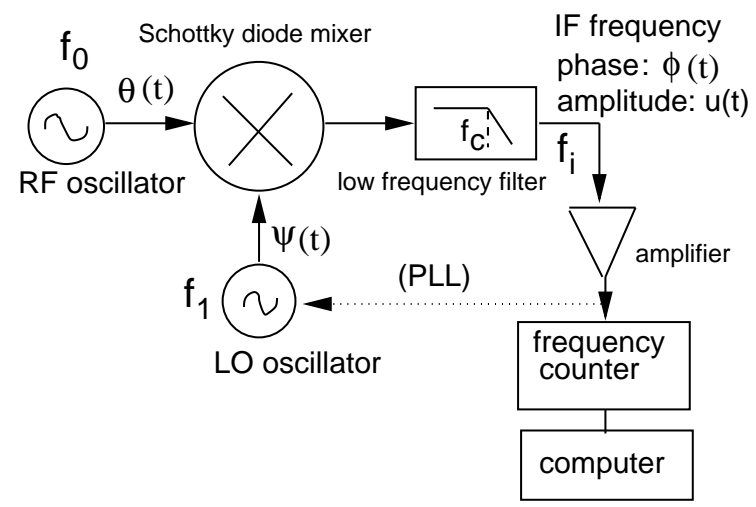

La boucle ouverte

Que fait ce montage? 
Si l'on suppose que le mélangeur effectue réellement le produit des deux signaux, nous obtenons en sortie du mélangeur un signal de la forme

$$
s(t)=\frac{a_{0}(t) a_{1}(t)}{2}\left(\cos \left(\left(f_{0}(t)+f_{1}\right) t\right)+\cos \left(\left(f_{0}(t)-f_{1}(t)\right) t\right)\right) .
$$

Supposons que $f_{0}(t)+f_{1}>f_{c}$ pour tout $t$, on a en appliquant le filtre passe-bas un signal de la forme

$$
s(t)=\frac{a_{0}(t) a_{1}(t)}{2} \cos \left(\left(f_{0}(t)-f_{1}(t)\right) t\right) .
$$

On voit que l'action du mélangeur idéal est linéaire en les fréquences et non linéaire en les amplitudes.

Malheureusement, un mélangeur réel a un comportement beaucoup plus compliqué. Par ailleurs, la modélisation des mélangeurs est loin d'être facile, même si par exemple, on connait exactement tous les composants électroniques qui le constitue. On renvoie à ([8],chapitre 12) pour plus de détails. Le mélangeur fait en général apparaître un spectre dit d'intermodulation ([8],p.314), i.e. l'ensemble des combinaisons à coefficients entiers entre $f_{0}$ et $f_{1}$ :

$$
p f_{0}-q f_{1}, \quad p, q \in \mathbb{Z}
$$

La structure du spectre des fréquences obtenu en sortie de la boucle ouverte reflète l'existence de ces modulations. La modélisation des composantes étant difficile il ne reste qu'une approche directe pour tenter de prédire et expliquer la structure du spectre des fréquences.

3.2. Résultats expérimentaux. — Le spectre des fréquences est de la forme suivante:

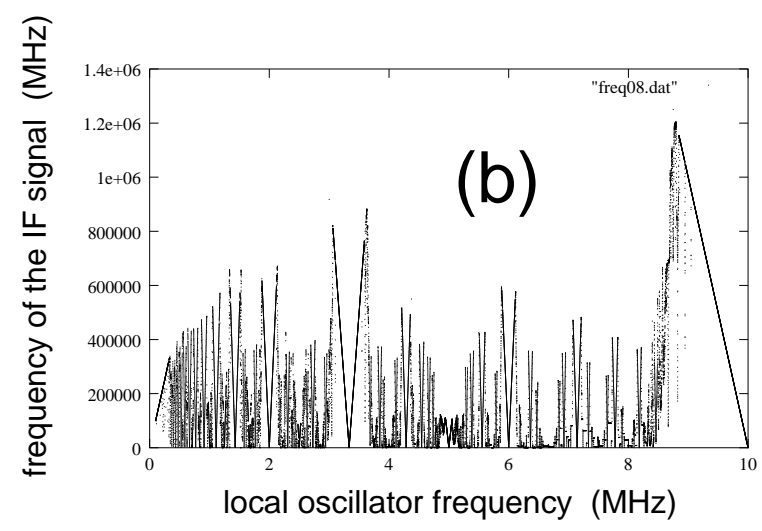

Spectre des fréquences

Les principaux traits de sa structure sont: 
- Les fréquences sont situées dans des bassins autour de fréquences rationnelles $p / q$, appellées zônes d'accrochage. Les bords de ces zônes sont notées $\nu^{-}(p / q)$ et $\nu^{+}(p / q)$.

- Les bassins ne sont pas symétriques. En effet, on observe que

$$
p / q-\nu^{-}(p / q) \neq \nu^{+}(p / q)-p / q .
$$

Dans la suite, nous allons développer une théorie quantitative qui rend compte de ces deux faits de manière précise.

\section{Spectre de fréquence théorique}

4.1. Formalisation et hypothèse diophantienne. - La principale différence entre le mélangeur idéal et le mélangeur "réel" est l'apparition d'harmoniques de la forme

$$
f_{p, q}(t)=p f_{1}-q f_{0}(t)
$$

$\operatorname{avec}(p, q) \in \mathbb{Z}^{2} \backslash\{(0,0)\}$.

L'action du filtre passe-bas de fréquence de coupure $f_{c}$ conduit à ne conserver que les harmoniques satisfaisant la relation

$$
\left|f_{p, q}(t)\right|<f_{c}
$$

Notons

$$
\nu(t)=\frac{f_{0}(t)}{f_{1}}
$$

la fréquence normalisée.

Le spectre des fréquences est donc gouverné par une équation du type

$$
\left|\nu(t)-\frac{p}{q}\right| \leq \frac{f_{c}}{q}
$$

Comprendre le spectre des fréquences, c'est déterminer les fréquences normalisées autorisées par la relation (8).

Nous allons pour un moment oublier l'aspect temporel (donc la dynamique de $\nu(t)$ ) et nous concentrer sur l'aspect statique du spectre des fréquences.

La première idée est que cette équation suffit à elle seule, à reconstruire le spectre des fréquences, ce qui est en soit un peu osé, car cela suppose une indépendance du spectre des fréquences vis à vis des amplitudes. 
Sans hypothèse sur la nature des approximations de $\nu$, on s'attend à trouver un bassin autour de chaque rationnel, bordé par deux segments de pente $\pm q$. C'est effectivement le cas. Malheureusement, cette approche ne permet pas de rendre compte de la dissymétrie des bassins observée expérimentalement.

Si l'équation (8) contient l'essentiel de l'information sur la nature du spectre de fréquence, c'est donc que l'approximation de $\nu$ n'est pas triviale. Il reste donc à déterminer la nature de l'approximation effectuée par le détecteur.

L'hypothèse que nous allons faire est que le détecteur a un comportement diophantien, i.e. que les approximations d'une fréquences $\nu$ sont effectuées par des convergents. Autrement dit, on doit vérifier la condition

$$
\left|\nu(t)-\frac{p_{i}}{q_{i}}\right| \leq \frac{f_{c}}{f_{0} q_{i}} \leq \frac{1}{\nu_{i+1} q_{i}^{2}}
$$

où $\nu=\left[\nu_{0}, \nu_{1}, \ldots\right]$ représente le développement en fraction continue de $\nu$, et $p_{i} / q_{i}=$ $\left[\nu_{0}, \ldots, \nu_{i}\right]$ est le $i$-ème convergent de $\nu$.

4.2. Principaux résultats. - Cette conditions a plusieurs conséquences, qu'il conviendra ensuite de vérifier expérimentalement:

i) Les fréquences $\nu$ observées en sortie du détecteur sont très contraintes par $(\mathrm{D})$. Soit $p / q$ un rationnel fixé, alors les $\nu$ associés ont un développement en fraction continue qui vérifie

$$
1 \leq \nu_{i+1}<\frac{f_{1}}{f_{c} q}
$$

ii) La condition (9) impose un seuil maximal pour $q$, à savoir

$$
q \leq\left[\frac{f_{1}}{f_{0}}\right] .
$$

Comme le dénominateur $q_{i}$ d'un convergent croit avec $i$, cela impose une profondeur maximale dans le développement en fraction continue de $\nu$.

Nous allons résumer la discussion précédente par le théorème suivant, qui pour un rationnel donné $p / q$, décrit l'ensemble des nombres réels admissibles sous la contrainte (8) et l'hypothèse diophantienne.

Théorème 1. - Soit $p / q$ une fraction irréductible. On note $S(p / q)$ l'ensemble des nombres réels satisfaisant (8) sous l'hyppothèse diophantienne (D). Alors, on a: 
- L'ensemble $S(p / q)$ est non vide si et seulement si $q \leq\left[f_{1} / f_{c}\right]$, où $[x]$ désigne la partie entière de $x$.

- Soit $\left[a_{1}, \ldots, a_{n}\right]$ le développement en fractions continues de $p / q$. L'ensemble $S(p / q)$ est l'ensemble des nombres réesl $x \in \mathbb{R}$ de développement en fractions continues $\left[x_{1}, \ldots, x_{k}, \ldots\right]$ tels que

$$
x_{i}=a_{i} \text { pour } i=1, \ldots, n,
$$

et

$$
x_{n+1} \leq f_{1} / f_{c} q
$$

Nous appelons spectre théorique des fréquences l'ensemble

$$
S_{f_{1} / f_{c}}=\{\nu \in \mathbb{R} \text { satisfaisant }(D)\} .
$$

Le théorème 11 permet de préciser la structure de $S_{f_{1} / f_{c}}$. C'est un théorème de nature prédictive puisqu'il permet de reconstruire le spectre des fréquences à partir de la donnée de $f_{1}$ et $f_{c}$. Afin de comparer les prédictions de notre théorie avec le spectre expérimental $\mathcal{S}_{f_{1} / f_{c}}$, nous démontrons le résultat suivant:

Lemme 1. - Soit $p / q$ une fraction irréductible, avec $q \leq\left[f_{1} / f_{c}\right]$ et $\left[a_{0}, a_{1}, \ldots, a_{n}\right]$ son développement en fraction continue. Le bord de la zône d'accrochage est donné par

$$
\begin{aligned}
\nu^{\sigma} & =\left[a_{0}, \ldots, a_{n}, a\right], \\
\nu^{-\sigma} & =\left[a_{0}, \ldots, a_{n}-1,1, a\right],
\end{aligned}
$$

avec $a=\left[f_{1} / f_{c} q\right]$ et $\sigma=+$ sin $n$ est pair et $\sigma=-$ si $n$ est impair.

La démonstration est donnée à la section 7.1.

Nous avons maintenant la caractérisation analytique des principaux éléments géométriques du spectre des fréquences théoriques.

4.3. Confirmation expérimentale. - On peut tester la validité de l'hypothèse diophantienne (D) via le théorème 1 et le lemme 11.

- Michel Planat et Serge Dos Santos [4] ont montré que les bords des zônes d'accrochage sont de la forme (14).

- L'hypothèse diophantienne prédit l'existence dans une zône d'accrochage donnée, d'une borne supérieure pour les quotients partiels $a_{\max }$. Cette borne se retrouve dans l'expression des bords de la zône d'accrochage. Michel Planat et Jean-Philippe Marillet [9] ont montré que $a_{\max }$ est bien de la forme $f_{1} / f_{c} q$. 
4.4. Exploration de la condition diophantienne. - Pour mieux comprendre la nature de l'hypothèse diophantienne $(\mathrm{D})$, nous allons procéder en deux étapes:

- La première consiste à comprendre l'effet d'une contrainte, que j'appellerai de résolution, sur les quotients partiels d'une fraction continue, qui découle du point i) ci-dessus. Autrement dit, nous allons regarder la structure des fractions continues à quotients partiels bornés par un $a_{\max } \in \mathbb{N}$ fixé. Cette étude, faite dans la prochaine section sera riche d'enseignement sur la différence essentielle qu'il existe entre travailler sur les nombres réels, et travailler sous une contrainte de résolution. En particulier, nous verrons que l'existence d'une résolution induit de fait une hiérarchie des nombres et donne naissance à une dynamique des nombres.

- La seconde étape prend en compte l'aspect dynamique du spectre des fréquences. La détection dans un bassin donné fait apparaître des sauts de fréquences. On donne ici une justification théorique de l'existence de ces sauts qui fait intervenir de manière essentielle l'hypothèse diophantienne.

\section{Espaces de résolution: aspects géométriques}

Ce paragraphe donne une construction géométrique de l'ensemble des fractions continues à quotient partiels bornés faisant apparaître une structure d'arbre. Cette construction n'est sans doute pas nouvelle, mais nous n'avons pas trouvé de référence faisant apparaître simplement les structures dont nous avons besoin. On renvoie au livre de G.H. Hardy et E.M. Wright ([6],p. 164-169) pour la présentation standard.

\subsection{Géométrie des fractions continues. -}

5.1.1. Représentation des fractions irréductibles. - Soit $p / q$ une fraction irréductible de $\mathbb{Q}$. On lui associe le point $(q, p) \in \mathbb{Z}^{2}$, ou de manière équivalente, la droite de $\mathbb{Z}^{2}$ passant par 0 et $(q, p)$, de pente $p / q$ et d'équation $q x-p y=0$.

On a donc une bijection entre $\mathbb{Q} \bigcup\{\infty\}$ et $P^{1}\left(\mathbb{Z}^{2}\right)$, l'ensemble des droites vectorielles de $\mathbb{Z}^{2}$, définie comme l'ensemble des points de $\mathbb{Z}^{2}$ modulo l'équivalence $(q, p) \sim\left(q^{\prime}, p^{\prime}\right)$ si et seulement si il existe un entier $\lambda \in \mathbb{Z}$ tel que $(q, p)=\lambda\left(q^{\prime}, p^{\prime}\right)$ ou $\left(q^{\prime}, p^{\prime}\right)=\lambda(q, p)$.

Chaque droite $D$ de $P^{1}\left(\mathbb{Z}^{2}\right)$ est isomorphe à $\mathbb{Z}$, et est engendré par un des deux points $(q, p),(-q,-p)$ de $D$ vérifiant $\left\langle q, p>=1\right.$. Ces points sont dit premiers dans $\mathbb{Z}^{2}$. On note $\mathcal{P}$ l'ensemble des points premiers de $\mathbb{Z}^{2}$. 


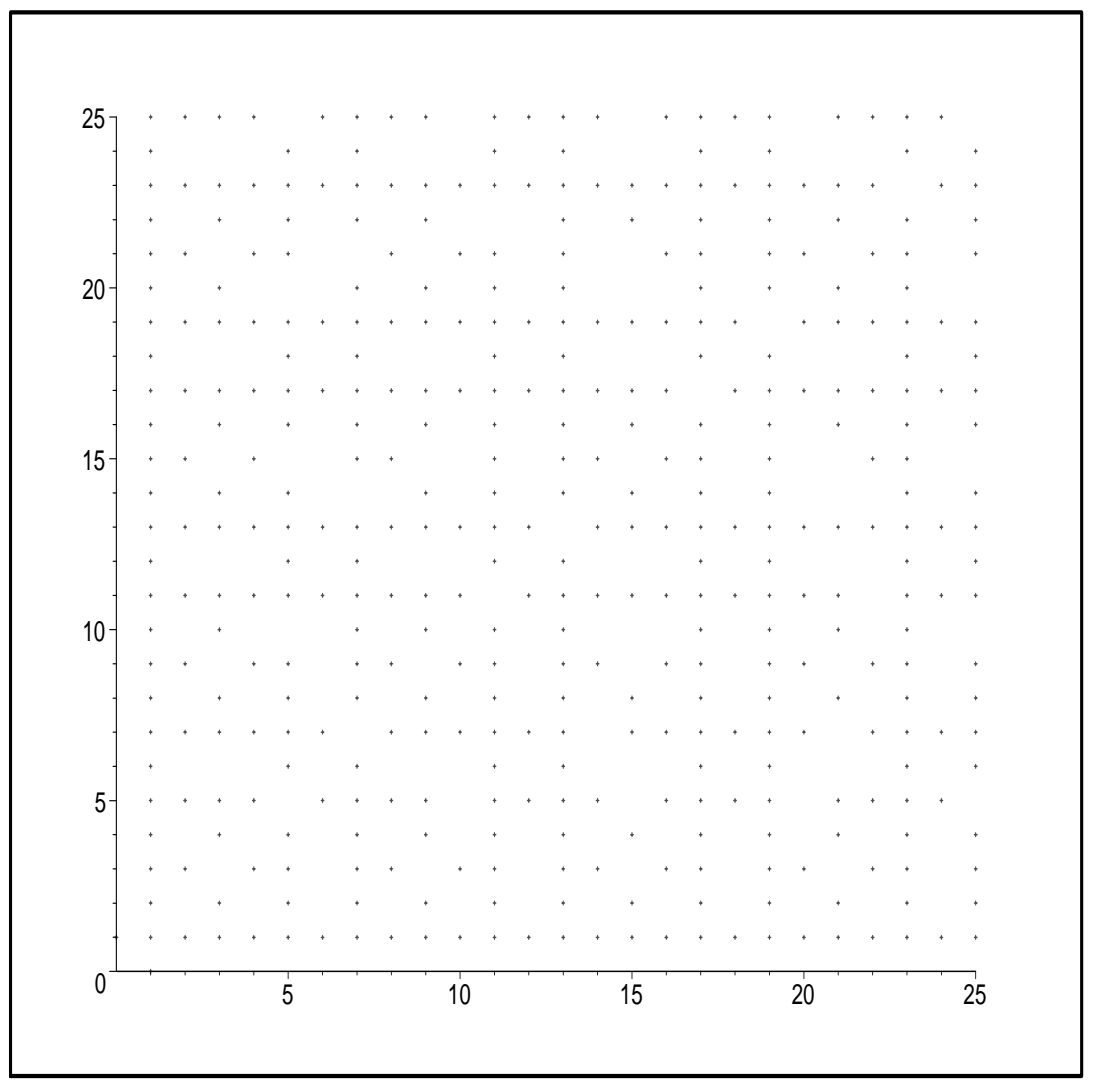

Figure 1. Points premiers de $Z^{2}(\operatorname{pgcd}(p, q)=1)$.

L'anneau des $\mathbb{Z}$-matrices $2 \times 2$, noté $M_{2}(\mathbb{Z})$, agit naturellement sur $\mathbb{Z}^{2}$ :

$$
\begin{aligned}
\forall A=\left(\begin{array}{ll}
a & b \\
c & d
\end{array}\right) \in M_{2}(\mathbb{Z}), & \mathbb{Z}^{2} \stackrel{A}{\rightarrow} \mathbb{Z}^{2}, \\
(q, p) & \mapsto(a q+b p, c q+d p) .
\end{aligned}
$$

Cette action induit une action sur $\mathbb{Q}$ via les transformations de Möbius:

$$
\begin{aligned}
A \in M_{2}(\mathbb{Z}), & \mathbb{Q} \\
z=p / q & \stackrel{A}{\rightarrow} \mathbb{Q}, \\
& \mapsto c z+d) /(a z+b) .
\end{aligned}
$$

La matrice $A$ préserve $\mathcal{P}$ si et seulement si $|\operatorname{det}(A)|=1$, i.e. $A$ est inversible dans $M_{2}(\mathbb{Z})$. On considère donc l'action de $G L_{2}(\mathbb{Z})$, l'ensemble des matrices inversibles de $M_{2}(\mathbb{Z})$, sur $\mathbb{Q}$, via les transformations de Möbius.

5.1.2. Fractions continues et $F_{2}^{+}$. — On renvoie à Khintchine [7] pour plus de détails. 
Soient $\left(a_{0}, \ldots, a_{n}\right)$ une suite finie d'entiers avec $a_{n} \neq 0$. On note $\left[a_{0}, \ldots, a_{n}\right]$ la fraction continue finie

$$
a_{0}+\frac{1}{a_{1}+\frac{1}{a_{2}+\frac{1}{\cdots+\frac{1}{a_{n}}}}}
$$

On conservera la même notation pour une suite de longueur infinie.

Supposons tous les $a_{i}>0$ pour $i>0$, alors tout irrationnel a une unique représentation comme fraction continue infinie. Par contre, l'égalité

$$
\left[a_{0}, \ldots, a_{n}\right]=\left[a_{0}, \ldots, a_{n}-1,1\right]
$$

montre qu'un rationnel possède deux écritures. On en déduit deux façon de rendre unique la représentation d'un rationnel :

i - tout nombre rationnel possède un unique développement en fraction continue de longueur pair (ou impair).

ii - tout nombre rationnel possède un unique développement en fraction continue se terminant par un entier $>1$.

La première représentation est adaptée à l'introduction du groupe modulaire. La seconde supprime les extensions virtuelles de la fraction continue via (18). Elle est bien adaptée à la construction de l'espace de résolution.

D'après l'algorithme des fractions continues, il est possible de construire toutes les fractions continues via les applications élémentaires de translation, notée $T$, et d'inversion, notée $S$ :

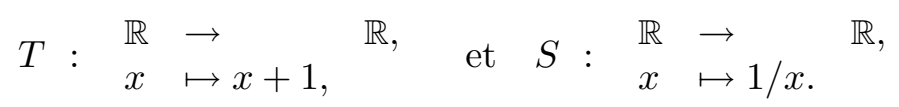

On peut restreindre l'action de $T$ (resp. $S$ ) à $Q$. Dans ce cas, on a deux homographies qui sont représentées dans $M_{2}(\mathbb{Z})$ par les matrices

$$
T=\left(\begin{array}{ll}
1 & 0 \\
1 & 1
\end{array}\right), \quad \text { et } \quad S=\left(\begin{array}{ll}
0 & 1 \\
1 & 0
\end{array}\right)
$$

Une fraction continue $\left[a_{0}, \ldots, a_{n}\right]$ s'écrit donc $T^{a_{0}} S T^{a_{1}} S \ldots S T^{a_{n}}(1,0)$. Pour obtenir une représentation unique, on choisi des représentations de longueur paire. On introduit la matrice $J=S T S$, de la forme

$$
J=\left(\begin{array}{ll}
1 & 1 \\
0 & 1
\end{array}\right)
$$

qui correspond à la transformation $x \rightarrow \frac{x}{x+1} \operatorname{sur} \mathbb{Q}$. 
On a :

Théorème 2. - Tout nombre rationnel $\left[a_{0}, \ldots, a_{2 n}\right]$ admet une unique représentation de la forme

$$
T^{a_{0}} J^{a_{1}} \ldots J^{a_{2 n-1}} T^{a_{2 n}}(1,0)
$$

Les matrices $T$ et $J$ sont unimodulaires (de déterminant 1). Elles engendrent le groupe modulaire $P S L_{2}(\mathbb{Z})$, qui est isomorphe au groupe libre de rang $2 F_{2}, T$ et $J$ étant deux générateurs libres. On note $F_{2}^{+}$le semi-groupe des mots écrit avec des puissances positives de $T$ et $J$. On a

\section{Corollaire 1. - L'application}

$$
\begin{array}{ll}
\mathbb{Q} & \rightarrow F_{2}^{+}, \\
{\left[a_{0}, \ldots, a_{2 n}\right]} & \mapsto T^{a_{0}} J^{a_{1}} \ldots J^{a_{2 n-1}} T^{a_{2 n},}
\end{array}
$$

est une bijection. Le groupe libre $F_{2}^{+}$agit à gauche sur $\mathbb{Q}$.

La démonstration découle du théorème précédent.

\subsection{L'arbre de Farey. -}

5.2.1. Terminologie sur les arbres. - On renvoie au livre de Serre ([19], §.2.2, p.28) pour plus de détails. On rappelle qu'un arbre est un graphe connexe, non vide, sans circuit. On adopte la convention suivante sur la représentation d'un arbre par un dessin : un point correspond à un sommet de l'arbre, et une ligne joignant deux points marqués correspond à une arêtes. Si l'arbre est orienté, une arête $\{P, Q\}$ étant donné, on appelle le sommet $P$, l'origine de $\{P, Q\}$ et $Q$ le sommet terminal de $\{P, Q\}$. Ces deux sommets sont les extrémités de $\{P, Q\}$.

Un sommet $P$ d'un arbre $\Gamma$ orienté étant donné, on appellera fils de $P$ l'ensemble des sommets terminaux des arêtes ayant $P$ comme origine. On appellera père de $P$, l'origine de l'arête ayant $P$ comme sommet terminal.

5.2.2. Arbre de Farey. - Habituellement, on représente l'arbre de Farey via l'action du groupe modulaire sur le demi-plan de Poincaré (ou de manière équivalente sur le disque de Poincaré). On en donne ici une représentation dans $\mathbb{Z}^{2}$, plus commode pour la suite.

On note $L_{\infty}$ la droite passant par $(1,0)$, engendré par l'action de $T$ sur le segment $[(1,0),(1,1)]$. Elle est de pente $\infty$.

De même, on note $L_{0}$ la droite passant par $(0,1)$, engendré par l'action de $J$ sur le segment $[(0,1),(1,1)]$. Elle est de pente 0 .

L'action de $F_{2}^{+}$sur $\mathbb{Z}^{2}$, induit une action de $F_{2}^{+}$sur $L_{0}$ et $L_{\infty}$. On obtient la figure suivante : 


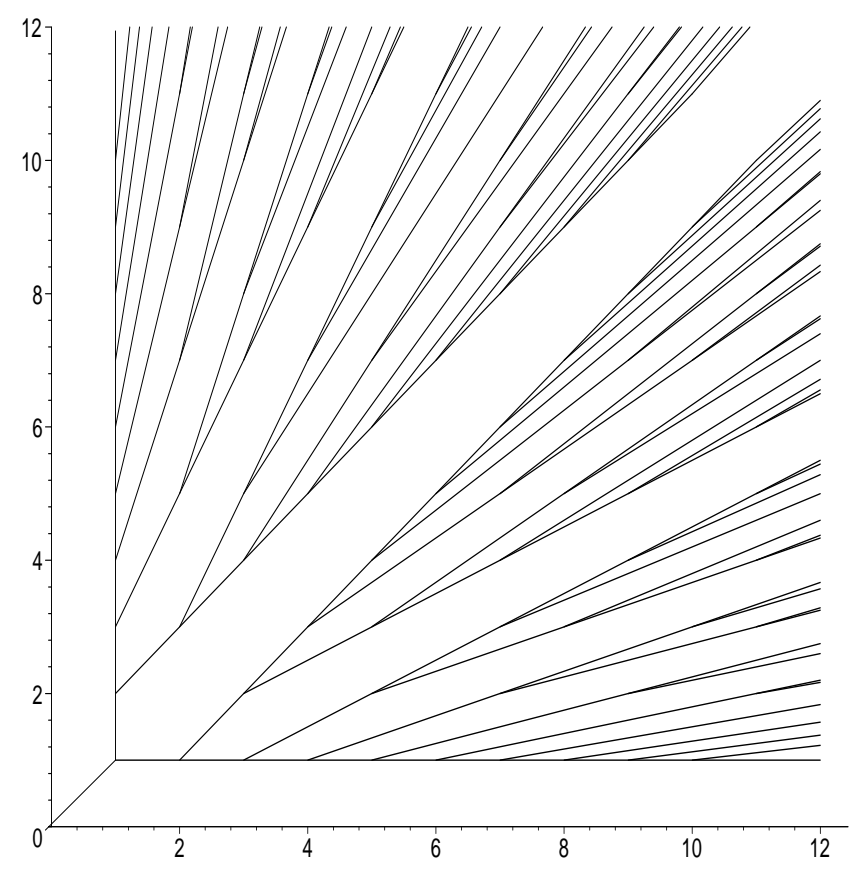

Figure 2. L'arbre de Farey

On note $\mathcal{T}$ l'ensemble ainsi obtenu. On a :

Théorème 3. - L'ensemble $\mathcal{T}$ est un arbre (ou plutôt, la réalisation geométrique d'un arbre) dont les sommets sont les points $(q, p) \in \mathbb{Z}^{2}$ irréductibles.

Ce résultat est classique (au moins dans le demi-plan de Poincaré, voir ( $\mathbf{1 9}$,§.4.2,p.52$53)$ ).

Nous allons préciser, la relation entre le développement en fraction continue d'un sommet de $\mathcal{T}$, celui de ses fils et de son père. Pour cela, nous introduisons la notion de branches et rameaux de l'arbre $\mathcal{T}$.

Définition 1. - Une branche de $\mathcal{T}$ est l'image par un mot de $F_{2}^{+}$des droites $L_{0}$ ou $L_{\infty}$. Soit $B$ une branche de $\mathcal{T}$, on appellera rameau de $B$ en $P$, une branche distincte de $B$ ayant pour origine le sommet $P$.

Une conséquence du théorème précédent est : 
Corollaire 2. - Soit $M=(q, p) \in \mathbb{Z}^{2},(q, p) \neq(1,1)$, un sommet de $\mathcal{T}$, alors $M$ appartiens à deux branches distinctes $B_{M}^{m}$ et $B_{M}^{f}$, appelées branche mère et fille. La branche mère admet la branche fille comme rameau en $M$.

La caractérisation de l'arbre $\mathcal{T}$ en terme de fractions continues s'énonce maintenant comme suit :

Théorème 4. - Soit $M=(q, p)$ un sommet de $\mathcal{T}$, tel que $p / q=\left[a_{0}, \ldots a_{2 n}\right]$. On note $B_{M}^{m}$ et $B_{M}^{f}$ ses branches mère et fille respectivement. On a :

$i$ - l'origine de la branche mère est

$$
\begin{array}{lll}
{\left[a_{0}, \ldots, a_{2 n-1}, 1\right]} & \text { si } & a_{2 n}>1 \\
{\left[a_{0}, \ldots, a_{2 n-2}+1\right]} & \text { si } & a_{2 n}=1 .
\end{array}
$$

ii - la pente de la branche mère est

$$
\begin{array}{lll}
{\left[0, a_{0}, \ldots, a_{2 n-1}-1,1\right]} & \text { si } & a_{2 n}>1, \\
{\left[0, a_{0}, \ldots, a_{2 n-2}\right]} & \text { si } & a_{2 n}=1 .
\end{array}
$$

iii - La pente de la branche fille est

$$
\begin{array}{lll}
{\left[a_{0}, \ldots, a_{2 n}-1\right]} & \text { si } & a_{2 n}>1 \\
{\left[a_{0}, \ldots, a_{2 n-1}\right]} & \text { si } & a_{2 n}=1 .
\end{array}
$$

Démonstration. - Elle repose sur la construction itérative de l'arbre de Farey.

i) La branche mère de $M$ est l'image par un mot de $w \in F_{2}^{+}$de la droite $L_{0}$ ou $L_{\infty}$.

Supposons que $B_{M}^{m}$ soit l'image de $L_{\infty}$ par $w$ (le cas de $L_{0}$ se démontre de la même manière). L'origine de $B_{M}^{m}$ est donc $w(1,0)$. Si $M \neq w(1,0)$, il existe un entier $k>0$ tel que

$$
M=w T^{k}(1,0)=T^{a_{0}} \ldots \ldots T^{a_{2 n}}(1,0),
$$

ou $w T^{k}$ est le mot obtenu par concaténation de $w$ et $T^{k}$.

Comme le mot $w$ ne se termine pas par un $T^{l}, l>0$, on déduit de (24) et de l'unicité de l'écriture de $M, k=a_{2 n}$ et $w=T^{a_{0}} \ldots J^{a_{2 n-1}} T$ si $a_{2 n}>1$, d'où l'origine de la branche mère dans ce cas est $w(1,0)=\left[a_{0}, \ldots, a_{2 n-1}, 1\right]$.

Si $a_{2 n}=1$, on écrit $\left[a_{0}, \ldots, a_{2 n-1}, 1\right]=\left[a_{0}, \ldots, a_{2 n-1}+1\right]$, d'où $M=w T^{a_{2 n-1}}(1,0)$, avec $w=T^{a_{0}} B^{a_{1}} \ldots T^{a_{2 n+2}+1}$. On a donc l'origine de la branche mère donnée par $w(1,0)=$ $\left[a_{0}, \ldots, a_{2 n+2}+1\right]$.

ii - Il suffit de noter que $L_{0}$ (resp. $L_{\infty}$ ) est paralléle à la droite passant par $(0,0)$ et $(1,0)$ (resp. $(0,0)$ et $(0,1))$. Quel que soit le mot $w \in F_{2}^{+}$, on a $w \cdot(0,0)=(0,0)$ car $w$ est une application linéaire. En utilisant i), la pente de la branche mère de $M=w \cdot(1,0)$, $w=T^{a_{0}} \ldots \ldots T^{a_{2 n}}$ est donc donnée par $T^{0} J^{a_{1}} T^{a_{2}} \ldots J^{a_{2 n-1}} T^{1}(1,0)$ si $a_{2 n}>1$ et par $T^{0} J^{a_{1}} \ldots, T^{a_{2 n-2}}(1,0)$ si $a_{2 n}=1$. 
iii - La démonstration est analogue à ii) en considérant la branche fille comme une branche mère d'origine $\left[a_{0}, \ldots, a_{2 n}\right]$.

5.3. Ensembles de résolution. - Le spectre des fréquences serait donné par l'ensemble précédent si aucune contrainte de résolution n'existait, i.e. dans un système idéal (au sens mathématique). Les résultats expérimentaux et la physique, imposent l'existence d'une résolution minimale. Dans ce paragraphe, nous interprétons cette contrainte et en donnons l'effet sur le spectre des fréquences.

5.3.1. La contrainte de résolution. - Il faut traduire la notion intuitive de résolution de manière à en obtenir une traduction simple sur l'ensemble des fractions continues.

- Hypothèse de résolution (nombres). Soit $a>0$ un entier. On identifie tout nombre réel $x \geq a$ à $\infty$.

On remarque que cette hypothèse de résolution à l'infinie implique, via l'action de l'application $S$, une condition de résolution en zéro. En effet, tous les nombres réels $0 \leq x \leq 1 / a$ sont identifiés à 0 .

On note $\mathcal{R}_{a}$ l'ensemble des nombres réels obtenus.

L' hypothèse se traduit sur les mots admissibles de $F_{2}^{+}$.

- Hypothèse de résolution (mots). Les seuls mots admissibles de $F_{2}^{+}$sont ceux ne contenant que des $T^{i}$ avec $i<a$.

On en déduit donc le théorème suivant :

Théorème 5. - L'ensemble de résolution $\mathcal{R}_{a}$ est l'ensemble des fractions continues à quotients partiels bornés.

Ce théorème n'apporte pas beaucoup à la compréhension de l'ensemble $\mathcal{R}_{a}$. Nous allons préciser la structure géométrique et dynamique de cet ensemble dans le prochain paragraphe.

\section{Espaces de résolution: aspects dynamiques}

6.1. Système dynamique de résolution. - On travaille maintenant dans $\overline{\mathbb{R}}^{+}=$ $\mathbb{R}^{+} \cup\{\infty\}$. Pour tout $a \in \mathbb{N}^{*}$, nous allons introduire une application naturelle sur $\overline{\mathbb{R}}$ appellée application de résolution.

Soit $a \in \mathbb{N}^{*}$, on note $F_{2}^{+}(a)$ l'ensemble des mots de $F_{2}^{+}$ne contenant pas de sous mots $T^{k}$ ou $J^{k}$ avec $k \geq a$. 
Définition 2. - Soit a $\in \mathbb{N}^{*}$, w un mot fini de $F_{2}^{+}, w=w_{1} \ldots w_{n}$, on définit l'application de $\mathrm{F}_{2}^{+}$dans $\mathrm{F}_{2}^{+}$qui ̀̀ $w$ associe $w_{a}$ obtenu en remplaçant le premier $T^{i}$ ou $J^{i}$ avec $i \geq$ a par $\infty$ ou $O$ respectivement. On note $R_{a}$ cette application.

L'application $R_{a}$ défini un système dynamique sur $F_{2}^{+}$. L'ensemble invariant maximal de $R_{a}$ est $F_{2}^{+}(a)$. La traduction sur les nombres se fait via l'application

$$
\begin{aligned}
& r_{a}: \quad \overline{\mathbb{R}}^{+} \quad \rightarrow \quad \overline{\mathbb{R}}^{+}, \\
& x=w(1,0) \quad \mapsto \quad x_{a}=R_{a}(w)(1,0) .
\end{aligned}
$$

L'application $r_{a}$ défini un système dynamique sur $\overline{\mathbb{R}}^{+}$. Ce système dynamique est à ma connaissance nouveau. Son graphe est donné pour $a=3$ par:

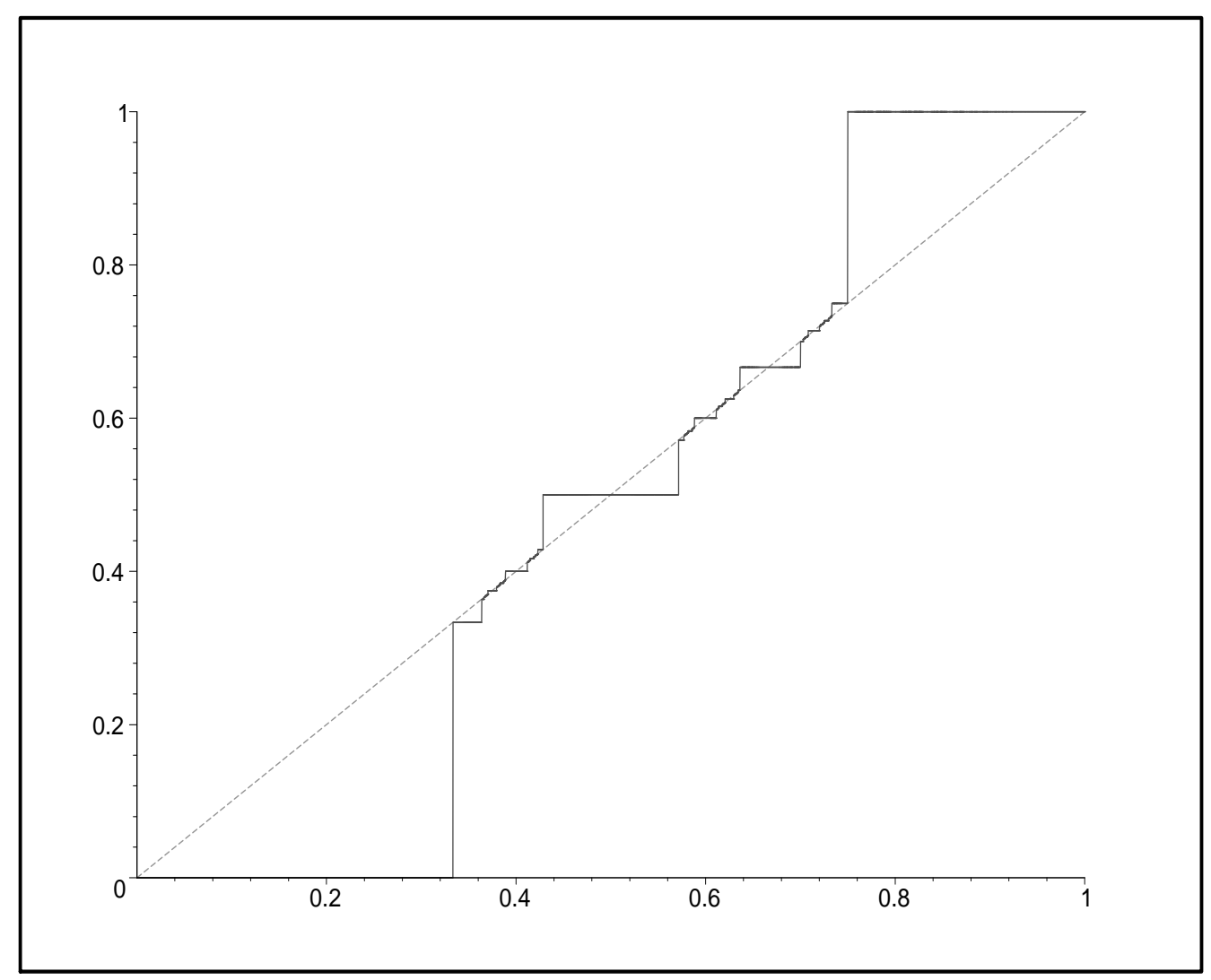

Figure 3. Graphe de l'application $r_{3}$.

L'ensemble invariant maximal de $r_{a}$ est $\mathcal{R}_{a}$.

Cette vision dynamique de l'ensemble des fractions continues à quotients partiels bornés permet de définir une dynamique naturelle des nombres. Précisons tout d'abord la structure géométrique de $\mathcal{R}_{a}$. 
6.2. Arbre de résolution. - Avant de formuler le théorème de structure sur $\mathcal{R}_{a}$, nous pouvons, en utilisant le procédé de construction utilisé pour l'arbre de Farey, construire l'ensemble $\mathcal{R}_{a}$ pour un $a$ fixé. Par exemple, dans le cas $a=3$, on a:

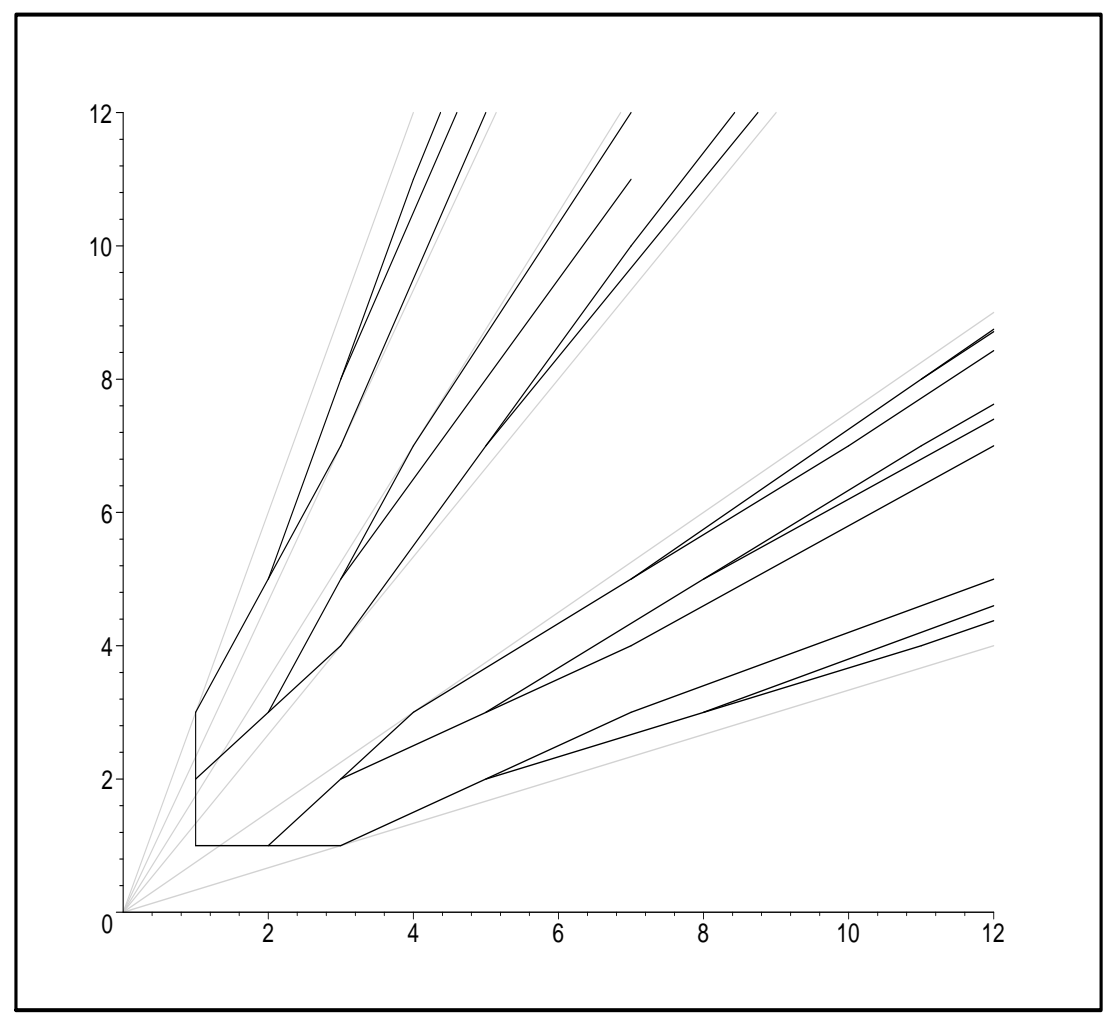

Figure 4. L'arbre de résolution $\mathcal{R}_{3}$.

Le principal effet de la contrainte de résolution est d'ouvrir les zônes du plan assoçiées à un rationnel donné. Par ailleurs, les nombres compris dans cette zônes sont envoyés par $r_{a}$ sur le rationnel correspondant au noeud.

\section{Construction dynamique et théorème de structure}

La construction précédente sur $\mathbb{Z}^{2}$ donne une vision géométrique qui n'est pas adaptée à une comparaison directe avec le spectre de fréquences expérimental. L'ensemble $\mathcal{R}_{a}$ peut se visualiser en traçant le graphe de la fonction notée $e_{a}: \mathbb{R} \rightarrow \mathbb{R}$ et définie par

$$
x \longmapsto\left|x-r_{a}(x)\right|,
$$

qui donne l'erreur d'approximation.

On obtient la figure suivante: 


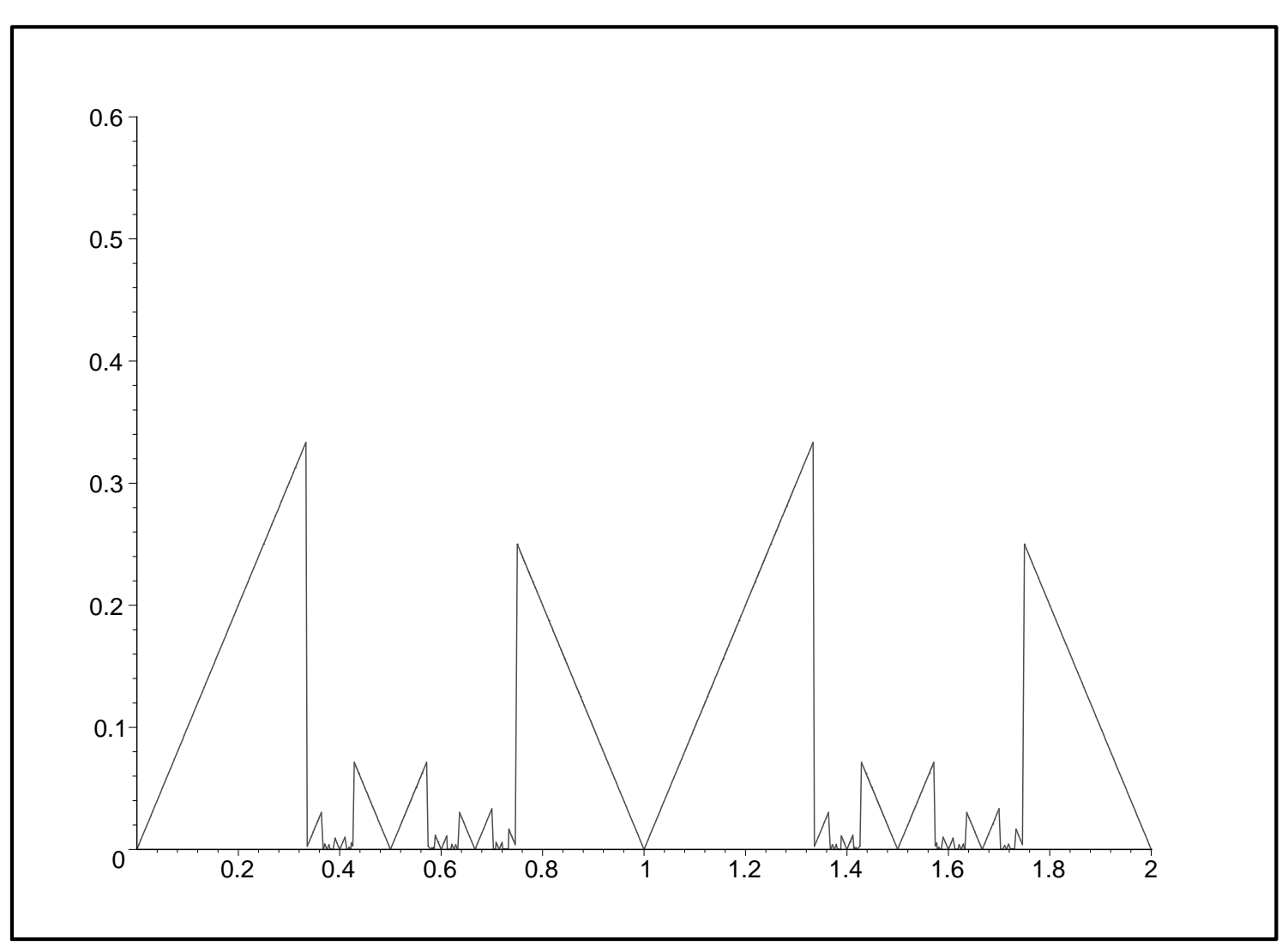

FiguRE 5. Graphe de l'erreur d'approximation pour $a=3$

On peut aussi la faire "à la main" de manière itérative, en transportant la première structure qui apparait, à savoir la zône d'accumulation au voisinage de zéro, et en regardant ce que devient cette structure via les opérations $x \mapsto x+1$ et $x \mapsto 1 / x$. Le traçé de la fonction d'erreur d'approximation apparait ainsi tout seul. Il montre aussi comment la première zône d'accrochage apparait au voisinage de 1 par transport de la zône d'accumulation en 0 via la translation et l'inversion laissant fixe le point 1 . Cette construction à l'avantage d'être simple et parlante.

Nous avons le théorème de structure suivant :

Théorème 6. - Soit a $\in \mathbb{N}^{*}$, l'ensemble de résolution $\mathcal{R}_{a}$ se décompose en:

$i$ - rationnels attractifs : soit $p / q$ un tel rationnel, il défini un interval d'accrochage $I_{p / q}^{a}=\left[\nu^{-}(p / q), \nu^{+}(p / q)\right]$ tel que pour tout $x \in I_{p / q}$, on a $r_{a}(x)=p / q$.

ii - rationnels transitoires : soit $p / q$ un tel rationnel, il défini un interval de transit à droite (resp. à gauche) $I_{p / q}^{+}=\left[p / q, \nu^{+}(p / q)\right]$ (resp. $I_{p / q}^{-}=\left[\nu^{-}(p / q), p / q\right]$ ) tel que pour tout $x \in I^{+}(p / q)$ (resp. $x \in I^{-}(p / q)$ ), on a $r_{a}(x)=p / q$. 
iii - irrationnels de blocage : ils sont obtenus comme accumulation de zones de blocage. $i v$-irrationnels transitoire : ils sont obtenus comme accumulation de zones de transit. $v$ - irrationnels mixtes : soit $\xi$ un tel irrationnel. Il est obtenu comme accumulation de zones de transit et de blocage.

Ce théorème n'est qu'une retraduction du fait que l'ensemble de résolution $\mathcal{R}_{a}$ est un arbre. On peut aussi le voir directement via la construction itérative de $\mathcal{R}_{a}$.

7.1. Sur les zônes d'accrochages. - Dans ce paragraphe, on travaille dans un ensemble de résolution donné $\mathcal{R}_{a}, a \in \mathbb{N}^{*}$.

Soit $x=p / q$ un rationnel de blocage. On a

Lemme 2. - Pour tout nombre rationnel de blocage $x=p / q \in \mathcal{R}_{a}$, on a

$$
\begin{aligned}
i-\nu^{\sigma}(1+x) & =1+\nu^{\sigma}(x), \quad \sigma= \pm, \\
i i-\nu^{\sigma}(1 / x) & =\frac{1}{\nu^{-\sigma}(x)} .
\end{aligned}
$$

Ces relations gardent un sens pour tout nombre $x \in \mathbb{R}^{*}$, ce qui permettra de ne plus préciser si on travaille avec un rationnel de blocage.

Lemme 3. - Soit $p / q=\left[a_{0}, \ldots, a_{n}\right] \in \mathcal{R}_{a}$, on a

$$
\begin{aligned}
\nu^{\sigma} & =\left[a_{0}, \ldots, a_{n}, a\right], \\
\nu^{-\sigma} & =\left[a_{0}, \ldots, a_{n}-1,1, a\right],
\end{aligned}
$$

avec $\sigma=+$ si $n$ est pair et $\sigma=-$ si $n$ est impair.

Démonstration. - On fait la démonstration pour $\nu^{+}$, la démarche étant analogue pour $\nu^{-}$. On a

$$
\nu^{+}\left(\left[a_{0}, \ldots, a_{n}\right]\right)=\nu^{+}\left(a_{0}+\frac{1}{\left[a_{1}, \ldots, a_{n}\right]}\right)=a_{0}+\nu^{+}\left(\frac{1}{\left[a_{1}, \ldots, a_{n}\right]}\right),
$$

par l'égalité i) du lemme 2. De plus, on a

$$
\nu^{+}\left(\frac{1}{\left[a_{1}, \ldots, a_{n}\right]}\right)=\frac{1}{\nu^{-}\left(\left[a_{1}, \ldots, a_{n}\right]\right)},
$$

par ii). Une simple récurrence donne donc

$$
\nu^{+}\left(\left[a_{0}, \ldots, a_{n}\right]\right)=\left[a_{0}, \ldots, a_{n-1}, \nu^{\sigma}\left(a_{n}\right)\right],
$$

avec $\sigma=+$ si $n$ est impair et $\sigma=-$ sinon.

Comme on a pour tout entier $0<m<a, \nu^{+}(m)=m+\frac{1}{a}, \nu^{-}(m)=m-1+\frac{1}{1+\frac{1}{a}}$ et de plus, $\nu^{+}(0)=1 / a, \nu^{-}(a)=a-1+\frac{1}{1+\frac{1}{a}}$, on en déduit le lemme. 
Ce résultat est le plus frappant vis à vis des données expérimentales. Les valeurs du bord des zônes d'accrochage prédites via ce lemme sont en accord quasi parfait avec celles obtenues expérimentalement (voir [4] et [9]).

7.2. Bassin d'attraction d'un rationnel. - Soit $a \in \mathbb{N}^{*}$ et $p / q$ un rationnel donné de $\mathcal{R}_{a}$. Le bassin d'attraction de $p / q$, noté $\mathcal{A}(p / q)$, est défini comme

$$
\mathcal{A}(p / q)=\left\{x \in \mathbb{R}, \exists k \in \mathbb{N}, r_{a}^{k}(x)=p / q\right\},
$$

où $r_{a}^{k}=r_{a} \circ \ldots r_{a} k$ fois.

Ces bassins sont formés de la zône d'accrochage proprement dire et des zônes transitoires accolées.

Avant de donner une caractérisation du bord du bassin d'attraction, regardons un exemple ou $r_{a}$ agit non trivialement:

Soit $a=3$ et $p / q=[0,1,2,1,3]$. On a $r_{3}(p / q)=[0,1,2,1]=[0,1,3]$ et $r_{3}^{2}(p / q)=[0,1]$.

On voit donc ici un exemple de dynamique des approximations via l'application $r_{3}$. Ce phénomène est dû à l'existence de rationnels dont la fraction continue est de la forme

$$
\left[a_{1}, \ldots, a_{n}, a_{1}, 1, a\right] .
$$

Pour ces nombres l'action de $r_{a}$ ne donne pas de suite la bonne approximation. En effet, on a

$$
r_{a}\left(\left[a_{1}, \ldots, a_{n}, a_{1}, 1, a\right]\right)=\left[a_{1}, \ldots, a_{n}, a\right],
$$

soit

$$
r_{a}^{2}\left(\left[a_{1}, \ldots, a_{n}, a_{1}, 1, a\right]\right)=\left[a_{1}, \ldots, a_{n}\right] .
$$

L'évolution dynamique de l'approximation de $\left[a_{1}, \ldots, a_{n}, a_{1}, 1, a\right]$ s'arréte si et seulement si $a_{n}<a$. Le phénomène ci-dessus est à l'origine de la terminologie de rationnels transitoires dans le théorème de structure.

Le lemme suivant caractérise simplement le bord du bassin d'attraction d'un rationnel: Lemme 4. - Soit $a \in \mathbb{N}^{*}$ et $\left[a_{1}, \ldots, a_{n}\right]$ un rationnel donné de $\mathcal{R}_{a}$. Les bords de son bassin d'attraction sont des irrationnels quadratiques. Précisément, les valeurs des bords sont

$$
\left[a_{1}, \ldots, a_{n}, a-1,1, \ldots, a-1,1, \ldots\right] \text { et }\left[a_{1}, \ldots, a_{n}-1,1, a-1,1, \ldots, a-1,1, \ldots\right] .
$$


Démonstration. - La démonstration repose sur la construction itérative du bord de la zône d'accrochage en 1. On transporte ensuite ces bords pour obtenir le rationnel choisi. Nous allons faire la construction pour le bord droit du bassin d'attraction, le bord gauche n'offrant pas plus de difficultés.

Une zône transitoire étant donnée à droite de 1, on obtient la prochaine en appliquant les opérations suivantes: $x \mapsto 1 / x, x \mapsto x+a-1, x \mapsto 1 / x$ er $x \mapsto x+1$. Autrement dit, on itère l'application

$$
t_{a}(x)=1+\frac{x}{1+x(a-1)} .
$$

Les points fixes de cette fonction sont des irrationnels quadratiques. La forme de l'application $t_{a}$ traduite sur les fractions continues nous dit que ces irrationnels s'obtiennent en collant aux fractions continues du bord des zônes d'accrochage une suite infinie de $a-1,1$.

On peut étudier d'autres types de nombres irrationnels obtenus comme par exemple accumulation de zônes de blocage. On renvoie à ([3],p.317-318) pour un exemple. Néanmoins, ces résultats sont difficiles à tester et interpréter au niveau expérimental et physique.

\section{Approche dynamique du spectre des fréquences}

L'analyse précédente permet une reconstruction globale du spectre des fréquences, mais ne dit pas la manière dont les fréquences bougent au cours du temps lors de la détection du signal. Or, de récentes expériences de Michel Planat et Jean-Philippe Marillet [9] ont mis en évidence l'existence de sauts de fréquences au voisinage des résonances. Ce paragraphe donne une base théorique à ce phénomène fondée sur l'hypothèse diophantienne.

8.1. Dynamique des fractions continues. - Pour tout $\nu \in R \backslash \mathbb{R}$, on note $p_{i} / q_{i}$ son $i$-ème convergent. Pour chaque valeur de $i$, on regarde la zône d'accrochage attachée au rationnel $p_{i} / q_{i}$. La taille de cette zône est proportionnelle à $q_{i}$. Pour comprendre la dynamique des fractions continues sous l'hypothèse diophantienne on doit étudier l'évolution des $q_{i}$ lorsque $i$ croit.

8.2. Exposants de stabilité. — Il est possible de quantifier les variations de $q_{i}$ lorsque $i$ croit. Pour tout $i \geq 1$, il existe un unique réel $\tau_{i} \geq 1$ et $\gamma_{i}>0$ tel que

$$
q_{i+1}=\gamma_{i} q_{i}^{\tau_{i}}
$$


avec $1 \leq \gamma_{i}<q_{i}$. L'exposant $\tau_{i}$ peut se concevoir comme un exposant caractérisant la stabilité de $q_{i}$ lorsque $i$ croit.

L'analyse diophantienne fournit des renseignements intéressants sur cet exposant:

Lemme 5. - Soit $\nu \in \mathbb{R}$, ses exposants de stabilité sont uniformément bornés si et seulement si $\nu$ est un nombre diophantien.

Ce lemme découle du théorème de Siegel [20]: un nombre réel $\nu$ est diophantien si et seulement si il existe $\gamma>0$ et $\tau \geq 1$ tels que $q_{i+1} \leq \gamma q_{i}^{\tau}$.

On peut aussi étudier l'évolution des $q_{i}$ via la fonction de Brujno [2]:

La fonction de Brujno, notée $\mathcal{B}$, est définie pour tout $\nu \in \mathbb{R} \backslash \mathbb{Q}$, par

$$
\mathcal{B}(\nu)=\sum_{i \geq 0} \frac{\log q_{i+1}}{q_{i}}
$$

où $p_{i} / q_{i}$ est le $i$-ème convergent de $\nu$.

On renvoie au travail de S. Marmi, P. Moussa et J-C. Yoccoz 10 pour une étude détaillée des propriétés de cette fonction.

8.3. Instabilité au voisinage des résonances. - Commençons par un fait expérimental: lorsque $f_{0}=1.00000007 \mathrm{MHz}$ et $f_{1}=0.599975 \mathrm{MHz}$, on a

$$
\frac{f_{0}}{f_{1}}=0.599974958 \ldots=[0,1,1,2,1596,1,10, \ldots] .
$$

On observe que le détecteur effectue des sauts autour des valeurs suivantes de fréquence de battement:

$$
f=135,261,386 \mathrm{~Hz} \text {. }
$$

Comment comprendre ce phénomène ?

Pour tout $a \in \mathbb{N}^{*}$, on note $\nu(a)$ le nombre $[0,1,1,2, a]$ et $p(a) / q(a)$ son écriture sous forme de fraction irréductible. Si on note $f(a)$ la fréquence définie par

$$
f(a)=\left|p(a) f_{0}-q(a) f_{1}\right|,
$$

on obtient pour $a=1593,1594$ et 1595 les fréquences de battement 135, 261 et 386 respectivement. 
Autrement dit, les sauts de fréquences observées correspondent à des fluctuations des quotients partiels, en particulier du paramètre de troncature.

On peut comprendre cette situation de la façon suivante: lorsque le dénominateur du $i$-th convergent devient instable (i.e. lorsqu'on a une augmentation brusque du quotient partiel dans le développement en fraction continue), on a des zônes d'accrochage très fines. De ce fait, le système devient sensible aux perturbations.

\section{Réalité ou artefact ?}

La théorie que nous avons proposé n'explique pas pourquoi le système fait de l'approximation diophantienne. Il me semble que si une raison claire existe elle doit se trouver du coté de la physique microscopique et d'une compréhension plus fine de la physique des mélangeurs.

On peut aussi mettre en doute le fait que les effets de hiérarchie que nous avons observé sont dus au système physique et donc mettent en évidence finalement des propriétés de la nature. Cette suspicion tient au fait que nous n'avons pas accé à des données bruts. En effet, entre l'expérience proprement dite et les données se trouve un ordinateur pour l'acquisition et le traitement des données (il y a une phase de comptage sur le signal). Rien ne dit que la façon d'effectuer ce comptage et du même coup tout le traitement des données n'est pas finalement biaisé. Cette situation est inévitable et entre en fait dans tout procédé de mesure d'un système physique.

Je ne crois pas qu'il soit possible de trancher pour le moment. Il me semble que le problème est de même nature que celui de décider si le monde réel est un continuum ou discret. Je renvoie à la discussion de E. Schrödinger ([15],p.41-59) pour plus de détails.

Ce qui est sûr c'est que de nombreux problèmes de physiques font intervenir d'une manière ou d'une autre des résolutions, i.e. des limites à notre mesure du réel. Cette limitation n'a dans certains cas que peut d'incidence, comme dans l'étude de beaucoup de phénomènes macroscopiques. La précision toujours plus grande des mesures, notamment dans le cas des oscillateurs, nous fait toucher du doigt il me semble la structure infime du réel. On tombe alors sur des phénomènes nouveaux mais de portée universelle. Je renvoie encore une fois au texte de E. Schrödinger (15],p.49-59) ou, sur quelques pages, il donne une construction très proche dans l'esprit des espaces de résolutions pour démontrer les difficultés liées à l'hypothèse d'une nature continue. 


\section{Références}

[1] T. Apostol, Modular functions and Dirichlet series in number theory, Graduate Text in Math. 41, Springer-Verlag, 1976.

[2] A.D. Brujno, Analytical form of differential equations, Trans. Moscow Math. Soc. t. 25, 1971, $131-288$.

[3] J. Cresson, J-N. Dénarié, Geometry and dynamics of number under finite resolution, dans Noise, oscillators and algebraic randomness, Planat ed., Lect. Notes in Physics, 305-323, 2000.

[4] S. Dos Santos, Étude non linéaire et arithmétique de la synchronisation des systèmes: applications aux fluctuations de basse fréquence des oscillateurs ultra-stables, Thèse, Univ. FrancheComté, 1998.

[5] M.H. Edwards, Riemann's zeta function, Acad. Pres, New York, 1974.

[6] G. Hardy, E. Wright, An introduction to the theory of number, Oxford University Press, Amen House, London, 1965.

[7] A.Y. Khintchine, Continued fractions, P. Noordhoff Ltd, Groningen, 1963.

[8] T. H. Lee, The design of CMOS radio-frequency integrated circuits, Cambridge University Press, 1998.

[9] J-P. Marillet, Arithmétique de la détection de phase électronique, rapport de stage, IUT BelfortMotbeliard, 2000.

[10] S. Marmi, P. Moussa, J-C. Yoccoz, The Brujno functions and their regularity properties, Comm. Math. Physics, t. 186, 1997, 265-297.

[11] M. Planat, $1 / f$ frequency noise in a communication receiver and the Riemann hypothesis, dans Noise, oscillators and algebraic randomness, Planat ed., Lect. Notes in Physics, 2000.

[12] M. Planat, S. Dos Santos, N. Ratier, J. Cresson, S. Perrine, Close to resonance interaction of radiofrequency wawes in a Schottky diode mixer : $1 / f$ noise and number theory, dans Quantum noise and other low frequency fluctuations in electronic devices, P. Handel, A. Chung Eds, AIP Press, p. 177-187, 1999.

[13] M. Planat, S. Dos Santos, J. Cresson, S. Perrine, $1 / f$ frequency noise in a communication receiver and the Riemann hypothesis, dans ICNF 1999, $15^{e}$ International Conference on Noise in Physical systems and $1 / f$ fluctuations, 1999.

[14] M. Planat, J-P. Marillet, J. Cresson, Electronics of receivers and number theory, Poster, section Mathematical Physics, Third European Congress of Mathematics 2000, Barcelona.

[15] E. Schrödinger, Physique quantique et représentation du monde, Éditions du Seuil, Collection Points Sciences, 1992.

[16] C. Series, The modular surface and continued fractions, J. London Math. Soc. (2), 31 (1985), 69-80.

[17] C. Series, The geometry of Markhoff numbers, The mathematical Intelligencer, Vol. 7, (3) (1985), 20-29.

[18] J-P. Serre, A course in arithmetic, Graduate Text in Mathematics 7, Springer-Verlag, 1973.

[19] J-P. Serre, Arbres, Amalgames, SL $L_{2}$, Astérisque, 1972.

[20] C. Siegel, Iteration of analytic functions, Ann. Math., t. 43, 1942, 807-812.

[21] J. Smith, Modern communications circuits - 2nd ed., 1997.

[22] M. Waldschmidt, P. Moussa, J. M. Luck, C. Itzykson (Editor), From Number Theory to Physics, 1st ed. 1992. Corr. 2nd printing, 1995.

JACKY CRESSON, Université de Franche-Comté, Equipe de Mathématiques de Besançon, CNRSUMR 6623, 16 route de Gray, 25030 Besançon cedex, France. 